\title{
Magnitude of Aflatoxigenic Aspergillus Species, Level of Aflatoxin B 1, and Associated Factors in Stored Feed at Poultry Farms in Dire Dawa, Ethiopia
}

\author{
Ambachew Motbaynor (iD, Dawit Kassaye, Migbaru Keffale, and Pawlos Wasihun \\ Haramaya University, College of Veterinary Medicine, P.O.Box 138, Dire Dawa, Ethiopia \\ Correspondence should be addressed to Ambachew Motbaynor; ambachewmotbaynor@haramaya.edu.et
}

Received 8 December 2020; Accepted 11 August 2021; Published 21 October 2021

Academic Editor: Sumanta Nandi

Copyright ( 2021 Ambachew Motbaynor et al. This is an open access article distributed under the Creative Commons Attribution License, which permits unrestricted use, distribution, and reproduction in any medium, provided the original work is properly cited.

\begin{abstract}
Aflatoxin, the secondary toxic metabolite of Aspergillus species, particularly aflatoxigenic Aspergillus flavus and parasiticus, has a detrimental effect on poultry health and production. There exists some information gap about the magnitudes of aflatoxigenic Aspergillus species and aflatoxin in poultry feeds in the study area. Thus, the study was conducted to estimate the magnitude and assess the related potential factors of aflatoxigenic Aspergillus species with evaluations of the level of aflatoxin B1 in stored poultry feed at selected farms in Dire Dawa, Ethiopia. A cross-sectional study design was carried out on 374 poultry feed samples recruited by using a stratified simple random sampling technique. A pretested structured questionnaire was used to assess the level of knowledge and prevention practices associated with aflatoxin in poultry feed. The isolation of aflatoxigenic Aspergillus species was made by Aspergillus flavus parasiticus media, and aflatoxin B1 was estimated by aflatoxin B1 enzyme-linked immune sorbent assay. Results showed that the magnitude of aflatoxigenic Aspergillus species was $72.5 \%$ (95\% CI: 67.6-76.9). The odds at which the species isolated were higher $(p<0.05)$ in feeds stored more than two months (AOR $=2.69)$, the presence of rodents in the storing room $(\mathrm{AOR}=2.67)$, feeds having high moisture content $(\mathrm{AOR}=1.5)$, and feed ingredient types $(\mathrm{AOR}=4.3)$ compared to their counter parts. Only 34.4 and 32.8 percent of the respondents have better knowledge and apply prevention practice about fungal contamination and aflatoxin production in poultry feed, respectively. The occurrence of aflatoxigenic Aspergillus species in poultry feed was associated with the presence of rodents in the feed storing room with long storing period and high moisture contents of the feed. The knowledge and prevention practices employed by farm managers and workers about fungal contamination and aflatoxin in poultry feed are found low.
\end{abstract}

\section{Introduction}

The poultry sector continues to grow and industrialize in many parts of the world, and it is one of the key livestock subsectors of Ethiopia. Nowadays, poultry production is becoming commercially oriented as poultry enterprise requires small space or land allocation as compared to larger livestock types and crop enterprises. These large populations of commercially produced poultry rely on manufactured poultry feeds. However, the ingredients used for the manufacture of poultry feed are usually prone to aflatoxin contamination due to the environmental and storage conditions [1].
Aspergillus flavus (A. flavus) and Aspergillus parasiticus(A. parasiticus) are the widespread fungi isolated from a wide range of animal feeds and human foods, known to produce a highly carcinogenic, hepatotoxic, mutagenic, and immunosuppressive toxins called aflatoxins [2]. Aflatoxins are naturally occurring secondary metabolites of Aspergillus species that lead to global animal and public health problems acquired through the ingestion of fungi contaminated crops, animal feeds, and feed ingredients and through the accidental consumption of crop and crop products by humans. Especially in the tropical and subtropical regions of the world, the problem is more pronounced associated with the warm temperatures and 
humidity of the environment that favors the growth of the fungi [3].

Cereals and plant protein sources such as maize, wheat, soya bean, rice, wheat bran, and sunflower meals that are used in poultry feeds are the main sources of fungal contamination and aflatoxin production during preharvesting, harvesting, transportation, and storage [4]. Aspergillus germinates and grows better on feeds and grains at moisture levels of $15 \%$ or above with 25 to $35^{\circ} \mathrm{c}$ temperatures, but for maximum aflatoxin production, a moisture level above $17.5 \%$ and temperature of 27 to $30^{\circ} \mathrm{C}$ is required. Aflatoxins reduce the nutritional quality of ingredients by utilizing the nutrients present in the ingredients for their metabolism and propagation [5].

Aflatoxin B1 (AFB1) is the most prevalent and the greatest toxigenic threat from the four major types of the aflatoxin molecules, namely, B1, B2, G1, and G2 [6]. In poultry, AFB1 is rapidly absorbed from the small intestine into the mesenteric venous blood. B1 is metabolized into M1 and $\mathrm{B} 2$ in the liver, and the nicotinamide adenine dinucleotide phosphate-linked enzyme system reduces B1 and B2 to cyclopentanol and aflatoxinol in chicken. However, in laying chickens, aflatoxinol is the major metabolite in muscles and blood and both AFBland aflatoxinol accumulate in eggs [4]. AFB1 impairs all important production parameters in poultry including feed intake, feed conversion efficiency, weight gain, pigmentation, egg production, and male and female reproductive performance [7]. Hence, regular monitoring of AFB1 in poultry feeds is an essential prerequisite to prevent aflatoxin build-up in poultry feeds.

The effect of aflatoxin on poultry depends on the age, physiological status, health, nutrition status, and times of exposure. It is very difficult to get rid of or to reduce the contamination once the aflatoxin is produced because this toxin has a high physical and chemical stability. Poultry feed contains $60-80 \%$ grains mainly maize, rice, and wheat and their by-products. In developing countries such as Ethiopia, the best-quality grains are used for human consumption and grains of poor quality are used as animal feeds which increases the contaminations of the feeds by the toxin. There is an information gap about the magnitudes of aflatoxigenic Aspergillus species and the levels of AFB1 in poultry feeds in Ethiopia, but there are reports on grains such as maize, wheat, and sorghum [8] and in dairy feeds in and around Addis Ababa [9] which show high prevalence of the fungus and the toxin in the grains and feed ingredients. Hence, this research work aims to estimate the magnitude and assess the related potential factors of aflatoxigenic Aspergillus species with evaluations of the level of AFB1 in stored poultry feed at selected farms in Dire Dawa, Ethiopia, from November 2019 to January 2020. The findings of this study are expected to assist poultry enterprises, farmers, investors, associations, and unions by revealing the magnitudes of aflatoxigenic Aspergillus species and aflatoxins in poultry feed and recommending optimum management options to reduce the contaminations of aflatoxigenic Aspergillus species and aflatoxin production in the poultry feeds. In this study, the term feed ingredient is used to indicate any cereal and grains such as maize, wheat bran, soya bean, nug cake, and bone and blood meal used to formulate a concentrate poultry feed. Those concentrate poultry feeds that are formulated (mixed) by the farm themselves were considered as home-formulated (home mixed) feeds, whereas those formulated by the factory as bulk and distributed to the users were termed as commercially formulated feeds. From the separate assessment questions set for knowledge and preventive practice on mould contamination and aflatoxin, those individuals who scored $50 \%$ and above of the knowledge questions and the preventive practices were considered knowledgeable and applied preventive practices, respectively.

\section{Materials and Methods}

2.1. Study Area. The study was conducted at selected poultry farms in Dire Dawa city administration which is located 519 kilometers away from Addis Ababa. It is geographically located at $9^{\circ} 18^{\prime} 40^{\prime \prime} \mathrm{N} 42^{\circ} 07^{\prime} 26^{\prime \prime} \mathrm{E}$ and $950-1708$ meter above sea level (Figure 1). The minimum and maximum annual temperatures of the city were 27 and $32^{\circ} \mathrm{C}$, respectively, with an average humidity of $49.6 \%$. The mean annual rainfall is about $637-734 \mathrm{~mm} \mathrm{[10].} \mathrm{There} \mathrm{are} 34$ poultry farms in the study area; among these, 2 of them are broiler farms and 32 of them are layer farms which have $200-9000$ heads of hens per farm.

2.2. Source of Feed. The source of feed was all stored poultry feed from selected poultry farms at Dire Dawa city administration.

2.3. Study Design. A cross-sectional study was conducted to evaluate the magnitude and associated risk factors for the occurrence of aflatoxigenic Aspergillus species and to determine the level of AFB1 among feeds in the study area.

2.4. Sample Size Determination. To evaluate the magnitudes of Aspergillus species in the poultry feed (first objective), the sample size was determined by a single proportion formula using an online software, Open Epi Version 3 (http://www. openepi.com), taking the assumption of $95 \%$ level of confidence, $5 \%$ absolute precision, and the respective expected prevalence indicated in Table 1, and the largest sample size 374 was set for the first objective.

To assess the associated risk factors for the occurrence of Aspergillus species in poultry feed (second objective), the sample size was determined by a double proportion formula using an online software, Open Epi Version 3 (http://www. openepi.com), taking the assumption of $80 \%$ power, $95 \%$ level of confidence, and the expected proportion of aflatoxigenic Aspergillus species in different potential sources indicated in Table 2, and the largest sample size is 132 .

Therefore, to run the study simultaneously, the largest sample size from the two objectives was set, so 374 samples were required to undertake the study.

2.5. Sampling Techniques and Procedures. Comprehensive lists of poultry farms were obtained at the Dire Dawa city small-scale enterprise office to have the study frame. There 


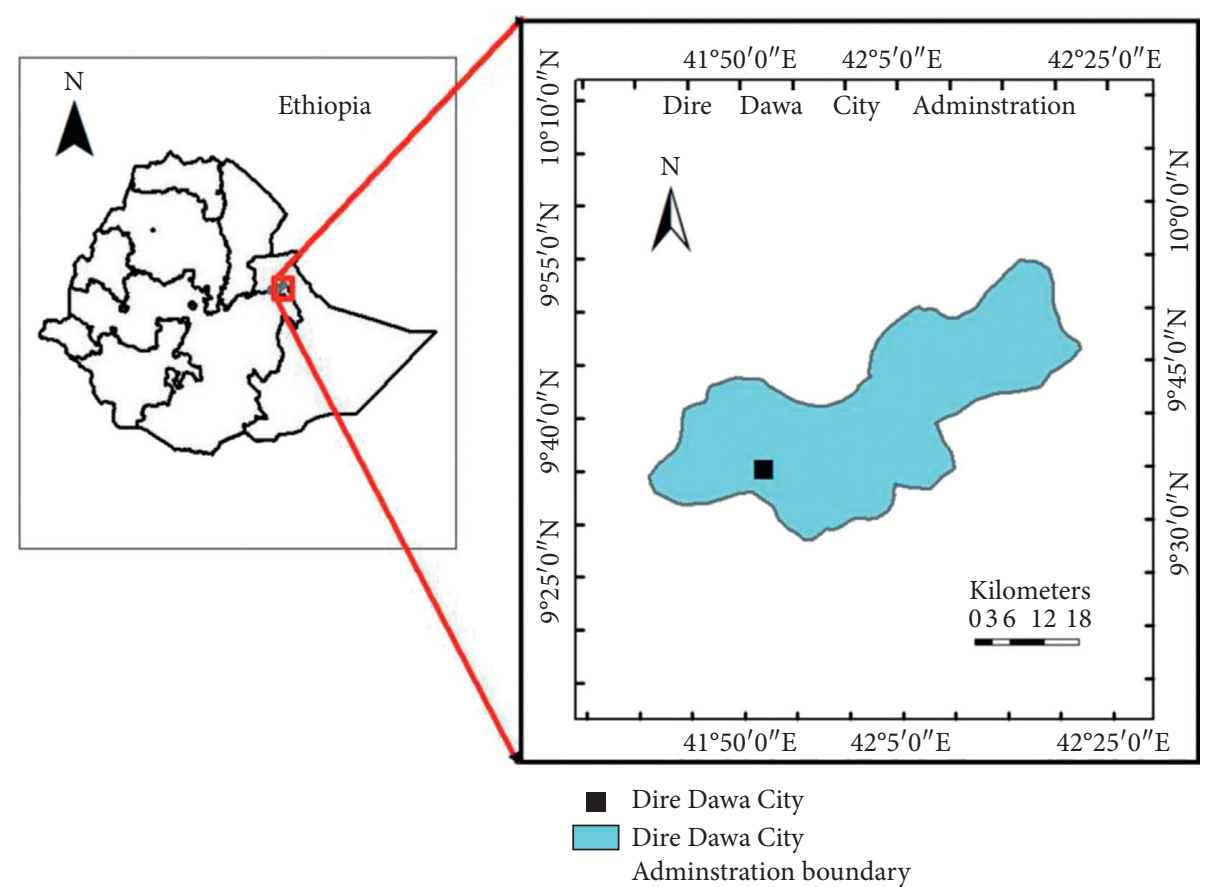

FIgURE 1: Dire Dawa city administration map.

TABLE 1: Required sample size for determining magnitudes of aflatoxigenic Aspergillus species.

\begin{tabular}{lccc}
\hline Study area & Magnitude (\%) & Calculated sample size & Reference \\
\hline Zaria, Nigeria & 42 & $\mathbf{3 7 4}$ & {$[11]$} \\
Cameroon & 83 & 217 & {$[12]$} \\
Kenya & 78 & 264 & {$[13]$} \\
\hline
\end{tabular}

Bold indicates the larger sample size from the three calculated sample sizes of the respective study area.

TABLE 2: Required sample size for different factors.

\begin{tabular}{lccc}
\hline Factors & Proportions (\%) in different groups & Calculated sample size & Reference \\
\hline \multirow{2}{*}{ Storage time } & Up to 30 days $(29.7 \%)$ & 66 & \\
& $31-90$ days $(66.6 \%)$ & 78 & {$[14]$} \\
Feed ingredient 1 & Noug cake $(58.3 \%)$ & & \\
& Wheat bran $(25 \%)$ & 132 & {$[15]$} \\
Feed ingredient 2 & Wheat bran $(4.4 \%)$ & & \\
\hline
\end{tabular}

were 32 poultry farms in the study area, and these farms were stratified based on the number of poultry flocks in to large-scale ( $\geq 1000$ heads of birds), medium-scale (500-999 heads of birds), and small-scale ( $<500$ heads of birds) farms to select participant farms. Sixteen (16) participant farms were selected randomly, and then, the sample size was allotted proportionally based on the number of sacks stored in each farm for each stratum (Figure 2). Then, the sample was taken by the systematic random sampling technique.

A semistructured questionnaire was designed and then administered to farm managers on the spot in the study farms. The questionnaire was designed to provide information on the knowledge and prevention practice of fungal contamination and aflatoxin production in the poultry feed at the farm level.
2.6. Sample Collection. $100 \mathrm{~g}$ sample per $100 \mathrm{~kg}$ of feed was collected at eight different portions of the lot (sacks) by using a sterile metal probe (Figure 3 ). Then, they were homogenized together, and $20 \mathrm{~g}$ of feed was taken from this homogenized feed by using a sterile spoon and divided into three parts: part one for moisture content determination, part two for isolations of aflatoxigenic Aspergillus species, and part three for AFB1 analysis. Part one samples were analyzed immediately to avoid change in moisture content, while parts two and three samples were stored in a refrigerator at $4{ }^{\circ} \mathrm{C}$ using a polythene bag until the day of analysis, which was never more than seven days after collection [16].

2.7. Moisture Content of the Feed. Determination of moisture feed content was performed within the same days of sample collection, by the oven method. Two grams of feed in a dish 


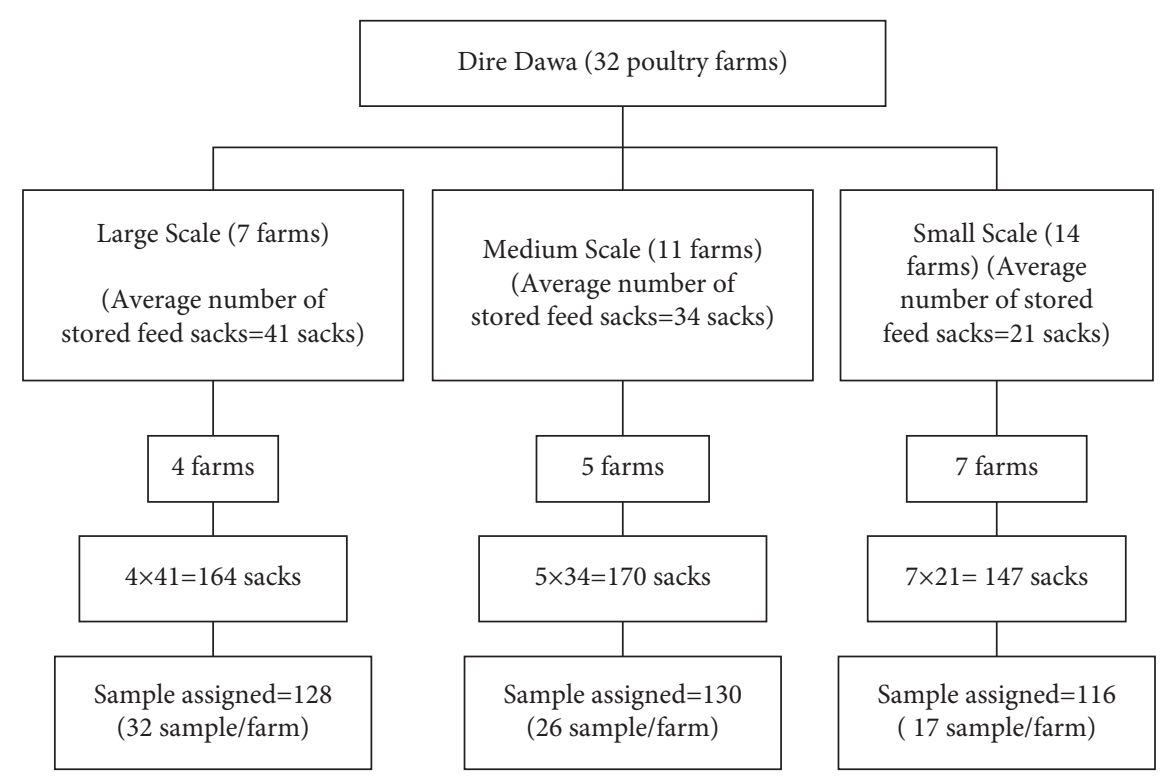

FIGURE 2: Schematic representation of the sampling procedure.

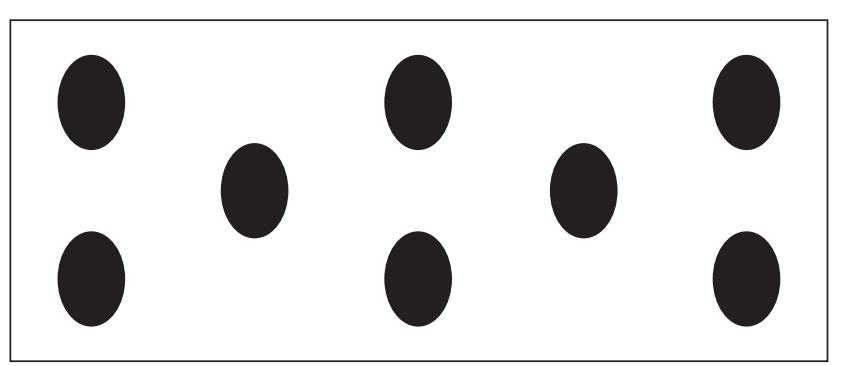

Figure 3: Sampling technique of feed on the lot.

was placed in an oven at $105^{\circ} \mathrm{C}$ overnight (16 hours). The feed was then weighed, and percentage moisture was calculated as tie percentage weight loss as follows:

$$
\% \text { moisture }=\frac{M_{o}-M_{1}}{M_{o}} \times 100,
$$

where $M_{o}=$ initial weight in grams; $M_{1}=$ final weight after drying in grams. Duplicate results for every sample were determined, and an average value was calculated per sample. The moisture contents of the feeds were categorized into three: low moisture content $(<10 \%)$, medium moisture content (10-14\%), and high moisture content (>14\%) [17].

\subsection{Isolation and Identification of the Aflatoxigenic Aspergillus Species}

2.8.1. Identification on Culture Media. Aflatoxin-producing Aspergillus species was isolated by Aspergillus flavus parasiticus agar media and prepared according to the manufacturer's instructions. One gram of the feed sample was aseptically transferred to a sterile test tube, and 9 milliliters of sterile distilled water was added to the feed and thoroughly mixed to perform a ten-fold serial dilution of the sample. Some $0.1 \mathrm{ml}$ of the dilution was cultured by a spread-plate technique using a sterile bent glass rod and then incubated at a temperature of $27^{\circ} \mathrm{C}$ for 72 hours. The aflatoxin-producing Aspergillus species developed intense yellow orange color at the base of the colonies which is a differential characteristic for these species. Then, the isolates were enumerated and subcultured on Sabouraud dextrose agar media at $27^{\circ} \mathrm{C}$ in the dark for 5 days to obtain pure cultures. Suspicious colonies of $A$. flavus were identified by their greenish-yellow appearance and powdery texture with the reverse side golden to red brown or pale to yellow, while $A$. parasiticus were identified by their blue-green appearance with the reverse side having white to yellow appearance [18].

2.8.2. Microscopic Identification. The microscopic features of the isolates were studied using the lactophenol cotton blue staining technique [19]. A drop of the stain was placed on a clean slide. A small part of the fungal cultures was removed and placed in the drop of the dye using a mounting needle. Then, the mycelium was spread by the same needle. Then, a cover slip was gently placed on the spread mixture with gentle digital pressure to eliminate air bubbles. Then, the slide was mounted and observed under an X40 objective lens. Identification of $A$. flavus was based on the presence of septate hyphae and rough and colorless conidiophores which end in a vesicle having the entire surface covered with either uni- or biseriate sterigmata (Phialid) and have bisereat metule, whereas $A$. parasiticus have unisereat metule and the vesicles are not covered entirely by uni- or biseriate sterigmata (Phialid) unlike A.flavus. Fungal taxonomic descriptions, identification keys, and Atlas were used as references [20].

2.8.3. Determination of Aflatoxigenic Aspergillus Species. In order to determine toxigenic Aspergillus species, the ammonia vapor test method was employed as described in [21]. The organism was centrally inoculated on potato 
dextrose agar (PDA) plates. The plates were incubated in the dark at a temperature of $27^{\circ} \mathrm{C}$ for 5 days. After 5 days of incubation, a set of plates were inverted over $2 \mathrm{ml}$ ammonium hydroxide for 15 minutes. Distilled water was used as a control instead of ammonium hydroxide. A change in color was used as a parameter to determine the toxigenic potentials of the Aspergillus species. Those strains having reverse turned pink color are recorded as positive (toxins producing strains). However, those without color change were recorded as negative (nontoxigenic strains).

\subsection{Determinations of Aflatoxin B1 in Feed and Feed Ingredients by the Competitive ELISA Technique}

2.9.1. Sample Preparation (Extraction) and Assay Procedure. Crushed and homogeneous solid samples were formed as a fine-to-medium-fine powder by using a crusher; then, the required amounts of the crushed sample were taken depending on the sample; the homogeneous sample was weighed in a flask and mixed with the required amounts of $80 \%$ methanol based on the sample. The suspension was shaken intensively for 3 minutes to extract the aflatoxin. The suspension was then filtered via Watman paper No 1 after settling the solids for quantitative analysis. The filtrate (sample extract) was diluted in a new container with a different ratio with the sample diluents depending on the samples [22].

All the reagents were brought to room temperature, and the PBS-Tween packet was reconstituted. We placed one mixing well in a microwell holder for each standard and the sample to be tested and placed an equal number of antibodycoated microtiter wells in another microwell holder and then dispensed the sample diluents into each mixing well; after that, we added each standard and the prepared sample to the appropriate mixing well containing diluents. We transfered contents from each mixing well to a corresponding antibody-coated microtiter well and incubated at room temperature; then, we added the substrate solution and incubated again; after that, we added the stop solution and finally read the optical density of each microwell [22].

2.10. Statistical Analysis. The data were entered in to Microsoft ${ }^{\circledR}$ Excel sheet for Windows 2010. Then, they were exported into STATA statistical software version 14.0 (STATA corp., College Station, TX, USA) for data processing and analysis. Binary logistic regression was used to measure the strength of the association between the response variable with each predictor variable, and odds ratio was determined. Multivariable logistic regression analysis was run to assess the strength of association between all predictor variables with the response variable. Variables with $p<0.2$ were selected as candidate to be included in the multivariate logistic regression analysis in an attempt to control for potential confounding variables, and adjusted odds ratio was determined. Colinearity between variables was also checked by standard error, and model fitness was assured by the Hosmor and Lemshow test. The AFB1 concentrations of the positive feed samples were calculated by using Graph Pad Prism 7 software. Throughout the data presentation, $P$ value less than 0.05 (i.e., $p<0.05$ ) was considered statistically significant.

\section{Results}

3.1. Magnitudes of Aflatoxigenic Aspergillus Species in Poultry Feed. Out of 374 poultry feed samples cultured, $72.5 \%$ (95\% CI, 67.6-76.9) were positive for aflatoxigenic Aspergillus species. From these, 48.9\% (95\% CI, 43.8-54.1) were A. flavus and the remaining $23.6 \%$ (95\% CI, 19.3-28.2) were contaminated with $A$. parasiticus. Among those samples with either of the species, $69.4 \%$ (95\% CI, 63.5-74.8) were toxigenic (Table 3).

From the feed types analyzed, high magnitudes of aflatoxigenic Aspergillus species contamination were shown in the mixed feeds $(81.7 \%)$ whereas low magnitudes were seen in wheat bran (35.6\%). High fungal contamination (81.6\%) was observed among feeds stored for more than 2 months, but low fungal contamination (58.1\%) was seen in feeds stored for less than a month. More fungal contamination $(92.7 \%)$ was seen in feeds having high moisture content $(>14 \%)$, while low fungal contamination $(35 \%)$ was seen in feeds with low moisture content $(<10 \%)$ (Table 4$)$.

3.2. Factors Independently Associated with Aflatoxigenic Aspergillus Species Contamination in Poultry Feed. Aflatoxigenic Aspergillus species contamination was found independently associated with the feed storing period, source of feed, presence of rodents in the feed storing room, moisture contents of the feed, and feed ingredient types $(p<0.05)$ (Table 5).

More specifically, the likelihood of aflatoxigenic Aspergillus species contamination in feeds stored one up to two months was nearly two times (AOR 1.99, 95\% CI: 1.05-3.76) that of those feeds stored less than one month. Feeds which were stored more than two months were 2.69 times (AOR 2.69, 95\% CI: $1.08-6.69)$ more likely to encounter aflatoxigenic Aspergillus species contamination than feeds that were stored less than one month. Feeds which were stored in rooms having rodents were 2.67 times (AOR 2.67, 95\% CI: 1.3-5.39) more likely to encounter aflatoxigenic Aspergillus species contamination than those which were stored in rodent-free rooms. Home-mixed (farm) feeds were $27 \%$ (AOR 0.73, 95\% CI: 0.39-1.1) less likely exposed to aflatoxigenic Aspergillus species contamination than commercially formulated feeds. The odds of fungal contamination in feed ingredients such as maize (AOR 4.3, 95\% CI: 1.6-11.5), soya bean (AOR 3.49, 95\% CI: 0.6-5.4), and nug seed cake (AOR 3.45, 95\% CI: 0.6-5.3) were more than three times those in wheat bran. Feeds which had a moisture content of less than $10 \%$ (low moisture content) were $81 \%$ (AOR 0.19, 95\% CI:0.1-0.25) less likely exposed to aflatoxigenic Aspergillus species contamination than feeds which had medium moisture content (10-14\%), whereas feeds which had high moisture content (>14\%) were 1.5 times (AOR 1.5, 95\% CI: 0.9-4.6) more likely to be exposed to aflatoxigenic Aspergillus contamination than those which had medium moisture content (10-14\%). However, aerations or 
TABle 3: Magnitudes of aflatoxigenic Aspergillus species and frequency distributions of $A$. flavus and A. parasiticus in poultry feed at Dire Dawa, from November 2019 to January 2020.

\begin{tabular}{lccccc}
\hline \multirow{2}{*}{ Aspergillus species } & \multicolumn{2}{c}{ Sample examined, $n=374$} & \multicolumn{2}{c}{ Toxigenic } & \multirow{2}{*}{ Toxigenic potential (\%) (95\% CI) } \\
& Sample positive & Magnitude (\%) $(95 \%$ CI) & Yes & No & $73.2(66.2-79.5)$ \\
A. flavus & 183 & $48.9(43.8-54.1)$ & 134 & 49 & $61.4(50.4-71.6)$ \\
A. parasiticus & 88 & $23.6(19.3-28.2)$ & 54 & 34 & $69.4(63.5-74.8)$ \\
Total & 271 & $72.5(67.6-76.9)$ & 188 & 83 & 83 \\
\hline
\end{tabular}

TABle 4: Magnitudes of aflatoxigenic Aspergillus species among the study variables in poultry feed at Dire Dawa, from November 2019 to January 2020.

\begin{tabular}{|c|c|c|c|c|c|}
\hline \multirow[t]{2}{*}{ Variables } & \multirow[t]{2}{*}{ Category } & \multicolumn{2}{|c|}{$\begin{array}{l}\text { Aflatoxigenic } \\
\text { Aspergillus } \\
\text { species }\end{array}$} & \multirow[t]{2}{*}{ Total } & \multirow[t]{2}{*}{ Magnitude (\%) (95\% CI) } \\
\hline & & Yes & No & & \\
\hline \multirow{3}{*}{ Feed storage period } & $<1$ month & 61 & 44 & 105 & $58.1(48.1-67.7)$ \\
\hline & $1-2$ month & 108 & 36 & 144 & $75.0(67.1-81.8)$ \\
\hline & $>2$ month & 102 & 23 & 125 & $81.6(73.7-88.0)$ \\
\hline \multirow{2}{*}{ Feed source } & Home mixed & 157 & 66 & 223 & $70.4(64.0-76.3)$ \\
\hline & Commercially formulated & 114 & 37 & 151 & $75.5(67.8-82.1)$ \\
\hline \multirow{2}{*}{ Room aeration } & Yes & 81 & 46 & 127 & $63.8(54.8-72.1)$ \\
\hline & No & 190 & 57 & 247 & $76.9(71.2-82.0)$ \\
\hline Presence of rodent & Yes & 220 & 57 & 277 & $79.4(74.2-84.0)$ \\
\hline In the store & No & 51 & 46 & 97 & $52.6(42.2-62.8)$ \\
\hline \multirow{6}{*}{ Feed ingredients } & Maize & 66 & 18 & 84 & $78.6(68.3-86.8)$ \\
\hline & Bone and blood meal & 18 & 12 & 30 & $60(40.6-77.3)$ \\
\hline & Soya bean & 29 & 10 & 39 & $74.4(57.9-87.0)$ \\
\hline & Wheat bran & 16 & 29 & 45 & $35.6(21.9-51.2)$ \\
\hline & Nug cake & 17 & 6 & 23 & $73.9(51.6-89.8)$ \\
\hline & Mixed feed & 125 & 28 & 153 & $81.7(74.6-87.5)$ \\
\hline \multirow{3}{*}{ Moisture content } & Low $(<10 \%)$ & 36 & 67 & 103 & $35(25.8-45.0)$ \\
\hline & Medium (10-14\%) & 146 & 29 & 175 & $83.4(77.1-88.6)$ \\
\hline & High $(>14 \%)$ & 89 & 7 & 96 & $92.7(85.6-97.0)$ \\
\hline
\end{tabular}

TABLE 5: Multivariate analysis results of associated factors for aflatoxigenic Aspergillus species contamination in stored poultry feed at farms in Dire Dawa, Ethiopia.

\begin{tabular}{|c|c|c|c|c|c|c|}
\hline \multirow[t]{2}{*}{ Variables } & \multirow[t]{2}{*}{ Category } & \multicolumn{2}{|c|}{$\begin{array}{c}\text { Fungal } \\
\text { contamination }\end{array}$} & \multirow[t]{2}{*}{ Total } & \multirow[t]{2}{*}{ AOR $(95 \% \mathrm{CI})$} & \multirow[t]{2}{*}{$p$ value } \\
\hline & & Yes & No & & & \\
\hline \multirow{3}{*}{ Storing } & $<1$ month & 61 & 44 & 105 & 1.00 & \\
\hline & 2 month & 108 & 36 & 144 & $1.99(1.05-3.76)$ & 0.034 \\
\hline & $>2$ month & 102 & 23 & 125 & $2.69(1.08-6.69)$ & 0.033 \\
\hline Moisture & Low $(<10 \%)$ & 36 & 67 & 103 & $0.19(0.06-0.25)$ & $<0.001$ \\
\hline \multirow{2}{*}{ Content } & Medium (10-14\%) & 146 & 29 & 175 & 1.00 & \\
\hline & High $(>14 \%)$ & 89 & 7 & 96 & $1.5(1.1-4.6)$ & 0.042 \\
\hline \multirow{2}{*}{$\begin{array}{l}\text { Source of } \\
\text { Feed }\end{array}$} & Home mixed & 157 & 66 & 223 & $0.73(0.39-0.99)$ & 0.001 \\
\hline & Commercially & 114 & 37 & 151 & 1.00 & \\
\hline \multirow{2}{*}{ Aeration } & No aeration & 81 & 46 & 127 & $1.7(0.9-3.26)$ & 0.1 \\
\hline & There is aeration & 190 & 57 & 247 & 1.00 & \\
\hline \multirow{2}{*}{ Rodent } & There is rodent & 220 & 57 & 277 & $2.67(1.3-5.39)$ & 0.006 \\
\hline & No rodent & 51 & 46 & 97 & 1.00 & \\
\hline \multirow[t]{3}{*}{ Feed } & Maize & 66 & 18 & 84 & $4.3(1.6-11.5)$ & 0.003 \\
\hline & Bone and blood meal & 18 & 12 & 30 & $1.87(0.89-3.5)$ & 0.25 \\
\hline & Soya bean & 29 & 10 & 39 & $3.49(1.6-5.4)$ & 0.031 \\
\hline \multirow{3}{*}{ Type } & Wheat bran & 16 & 29 & 45 & 1.00 & \\
\hline & Mixed feed & 125 & 28 & 153 & $6.3(3.25-16.2)$ & $<0.001$ \\
\hline & Nug cake & 17 & 6 & 23 & $3.45(0.6-5.3)$ & 0.008 \\
\hline
\end{tabular}


ventilation was not found to be an independently associated factor for aflatoxigenic Aspergillus species contamination in feed $(p<0.05)$ (Table 5).

3.3. Mean Aflatoxin B1 Levels of Mixed and Individual Ingredients of Poultry Feeds. From all mixed and individual ingredients of poultry feeds analyzed for AFB1, minimum and maximum mean AFB1 levels were $4.8 \mu \mathrm{g} / \mathrm{kg}$ and $31.2 \mu \mathrm{g} /$ $\mathrm{kg}$ for wheat bran and soya bean, respectively (Figure 4).

\subsection{Mean AFB1 Levels of Poultry Feeds and Daily Toxin Intake} of Hens with Respect to Feed Source. Based on the ration formulation that the farms used to prepare their poultry feeds described below, the minimum and maximum AFB1 level per $\mathrm{kg}$ of feed was $7.34 \mu \mathrm{g} / \mathrm{kg}$ and $41.0 \mu \mathrm{g} / \mathrm{kg}$ from home-mixed feeds and commercially formulated feeds, respectively, and the minimum and maximum AFB1 intake per hen per day were $0.92 \mu \mathrm{g}$ and $4.92 \mu \mathrm{g}$, respectively (Table 6).

$$
\begin{aligned}
& \text { Ration formulation per } 100 \mathrm{~kg} \text { of feed }=45 \% \text { maize } \\
& +16 \% \text { nug cake }+10 \% \text { soya bean } \\
& \quad+20 \% \text { bone and blood meal }+7.5 \% \text { wheat bran } \\
& \quad+0.5 \% \text { salt }+1 \% \text { vitamin. Total toxin intake/hen/day } \\
& \quad=(\text { daily feed intake } / \text { hen }) \\
& \text { * (total toxin in } \mu \mathrm{g} / 100 \mathrm{~kg} \text { of feed }) .
\end{aligned}
$$

Among home-mixed feeds, $75.0 \%$ were within the FDA AFB1 standard permissible limits $(\leq 20 \mu \mathrm{g} / \mathrm{kg})$ and only $25.0 \%$ exceeded the maximum FDA AFB1 permissible limits standards $(>20 \mu \mathrm{g} / \mathrm{kg})$, whereas in commercially formulated feeds, $62.5 \%$ of feeds fulfilled FDA permissible limits, and some $37.5 \%$ of the commercial feeds exceeded the maximum FDA permissible limits.

3.5. Knowledge Assessments of Farm Managers, Professionals, and Daily Poultry Farm Workers on Fungal Growth and Aflatoxin in Poultry Feeds. Sociodemographic characteristics among the farm managers, professionals, and daily farm workers were observed. From all the respondents, $62.5 \%$ were $20-30$ years old and $37.5 \%$ were females. Academically, $39.1 \%$ of the respondents were technical, vocational, and education training college (TVET) graduates and $28.1 \%$ were university graduates. From all the respondents, only $34.4 \%$ and $20.3 \%$ had knowledge about fungal growth and aflatoxin production in poultry feeds, respectively. Regarding the prevention practice, only $32.8 \%$ applied prevention practice on fungal contamination and aflatoxin production in poultry feeds (Table 7).

\section{Discussion}

The study showed that the magnitude of aflatoxigenic Aspergillus species contamination in poultry feed from poultry farms in Dire Dawa administrative council was $72.5 \%$. The finding is in agreement with $77.9 \%$ in Nigeria [23], 73.1\% in Pakistan [24], 65\% in Tanzania [25], 64.29\% in Sudan [26],

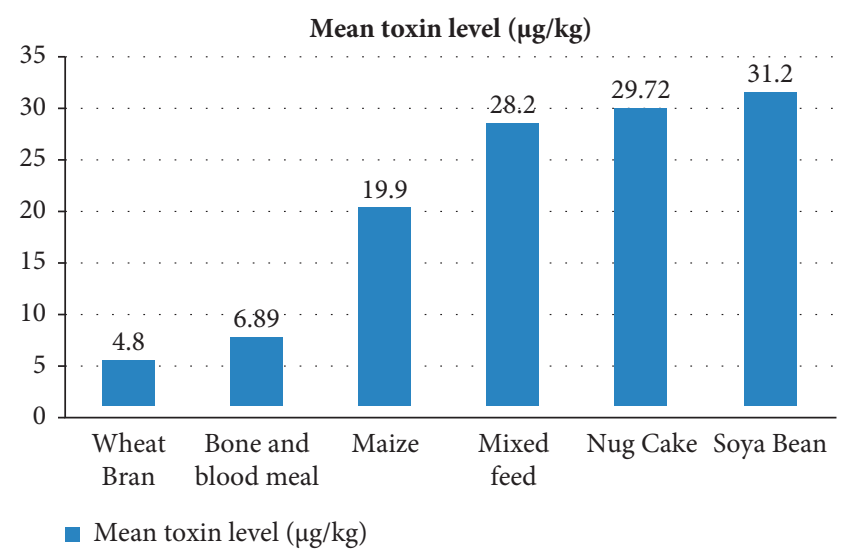

FIgURE 4: Mean AFB1 levels of stored poultry feed and feed ingredients in farms at Dire Dawa, Ethiopia.

and $57 \%$ in Myanmar [27], but it is lower than $83 \%$ in Cameroon [12]. However, it is higher than $46 \%$ in France [28], 35\% in Malawi [29], and 30\% in South Africa [30].

Low level of fungal contamination in this study as compared to Cameroon may be due to the environmental factors and the feed ingredient type used. Cameroon, where the studies were conducted, had an average temperature range of $22-300 \mathrm{c}$ and $60-80 \%$ humidity [12], which is a conducive environmental condition for fungal growth and aflatoxin production. In addition, maize and peanut are the main constituents of poultry feed in Cameroon [12] which are the most preferable substrates for fungal growth and aflatoxin production due to high contents of soluble sugars in maize and high lipid content in peanut [31], [32].

The high occurrence of fungal contamination in this study compared with South Africa and France may be due to the presence of well-developed commercial farming systems in France and South Africa [28, 33]), which reduces the fungal contamination and aflatoxin production by reducing the factors that promote the contamination of fungus and toxin production by pre- and postharvest crop management practices such as using crop hybrids that are less susceptible to fungal contamination and aflatoxin production, reducing physical damage to crops at harvest, pest management, good sanitation, and improved feed storing practices [34].

From the isolated Aspergillus species, $69.4 \%$ was aflatoxigenic. The result is comparable with $64.29 \%$ in Sudan [26] and 60\% in Pakistan [4], but it is lower than 98\% from Tamil Nadu, India [35], 88\% from cereals in Ethiopia [8], and higher than $42 \%$ in Zaria, Nigeria [11], 35\% in Malawi [29], and $16.1 \%$ in Ilaro, Nigeria [36]. These toxigenic variations may be due to variations in factors that determine the productions of aflatoxin in the respective countries such as temperature, humidity, storing periods, and moisture contents of feed together with the feed type they used.

From the feed ingredients, the maximum mean AFB1 was recorded from soya bean while the minimum mean AFB1 level was registered in wheat bran. This finding is comparable with that in Ethiopia [9, 37]. The maximum mean AFB1 in soya bean may be due to its high lipid content 
TABLE 6: Mean AFB1 level of home-mixed and commercially formulated poultry feeds.

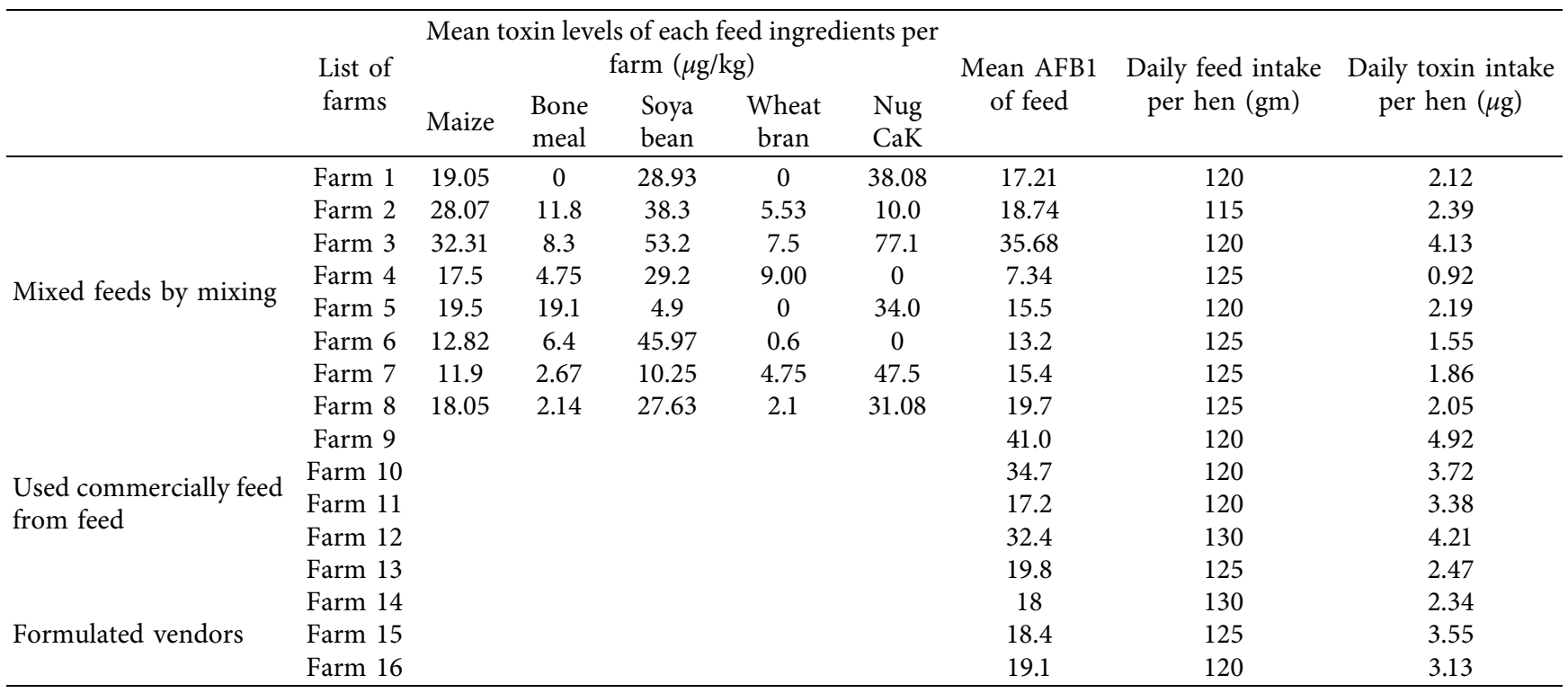

TABLE 7: Frequency distributions of sociodemographic, knowledge, and prevention practice assessments on fungal contamination and aflatoxin production in poultry feed.

\begin{tabular}{|c|c|c|c|}
\hline Variable & Category & Total & Percent (\%) \\
\hline \multirow{2}{*}{ Sex } & Male & 40 & 62.5 \\
\hline & Female & 24 & 37.5 \\
\hline \multirow{2}{*}{ Age (year) } & $20-30$ & 40 & 62.5 \\
\hline & Above 30 & 24 & 37.5 \\
\hline \multirow{3}{*}{ Education } & High-school certified & 21 & 32.8 \\
\hline & TVET graduate & 25 & 39.1 \\
\hline & University graduate & 18 & 28.1 \\
\hline \multirow{3}{*}{ Occupation } & Farm manager & 16 & 25 \\
\hline & Professionals & 35 & 54.7 \\
\hline & Daily farm worker & 13 & 20.3 \\
\hline \multirow{2}{*}{ Knowledge about fungal contamination } & Yes & 22 & 34.4 \\
\hline & No & 42 & 65.6 \\
\hline \multirow{2}{*}{ Knowledge about aflatoxin } & Yes & 13 & 20.3 \\
\hline & No & 51 & 79.7 \\
\hline \multirow{2}{*}{ Fungal contamination prevention practice } & Yes & 21 & 32.8 \\
\hline & No & 43 & 67.2 \\
\hline
\end{tabular}

which significantly stimulates fungal growth and AFB1 production [31], and the minimum mean AFB1 in wheat bran may be due to its low moisture content and poor nutritional composition as compared to other feed ingredient types [15].

In this study, concentrated (mixed) feeds were more exposed (81.7\%) to aflatoxigenic Aspergillus species contamination than feed ingredients. In feed ingredients, aflatoxigenic Aspergillus species contamination was seen as $78.6 \%$ in maize, $74.4 \%$ in soya bean, $73.9 \%$ in nug cake, $60 \%$ in bone and blood meal, and $35.6 \%$ in wheat bran as their exposing order. This finding is comparable with $80.9 \%$ in mixed feed, $65.3 \%$ in maize, $48.6 \%$ in soya bean, and $18.5 \%$ in wheat bran in India [38] but lower than $100 \%$ in mixed (concentrated) feed, $100 \%$ in maize, and $86.67 \%$ in soya bean at Bangladesh [39]. High fungal contamination in concentrated feed might be due to fact that concentrated feeds have high fat, carbohydrate, and protein content which is suitable for fungal growth and aflatoxin production [40]. From individual feed ingredients aflatoxigenic Aspergillus species contamination is high in maize and low in wheat bran. High fungal contamination in maize might be due to the presence of high soluble sugars (glucose, sucrose, and maltose) in maize which are excellent substrates for fungal growth [32]. Low fungal contamination and toxin production in wheat bran might be due to the fact that most wheat bran available as an animal feed is a by-product of wheat milling in the preparations of flour used for human consumption. In doing so, sorting and cleaning is carried out. Sorting and cleaning of grains to remove damaged ones is a process which has been shown to significantly reduce the aflatoxin level [32].

This finding showed that $25.0 \%$ of home-mixed feeds and $37.5 \%$ of commercially formulated feed exceeded the maximum FDA AFB1 permissible standard limits $(>20 \mu \mathrm{g} /$ 
$\mathrm{kg})$. The results are consistent with other studies conducted elsewhere [41, 42]. That more commercially formulated feeds exceeded FDA permissible limits than home-mixed feeds may be attributed to the fact that, in commercially formulated feeds, they are mixed during buying, thus creating favorable conditions for fungal contamination and aflatoxin production during transportation and storage, whereas in home-mixed feeds, the farms buy feed ingredients rather than mixed feeds and mix by themselves on farm; as a result, feeds are stored in the form of feed ingredients rather than mixed feeds and there may be cleaning and sorting feed ingredients during mixing. Therefore, preprocessing of ingredients helps to reduce fungal contamination and aflatoxin production in home-mixed feeds [43].

Based on this study, the minimum and maximum AFB1 intake per hen per day was $0.92 \mu \mathrm{g}$ and $4.92 \mu \mathrm{g}$, respectively, which was low. However, low doses of continues consumptions of AFB1 by the hen may have far-reaching effects on its health and overall performance. Since chronic exposure to small amounts of AFBlcauses morphological damage to small intestinal mucosal linings, the absorptive surface of the small intestine will decrease exposing the hens to feed deficiency, increasing disease susceptibility and vaccine failure [15].

Among the aflatoxigenic Aspergillus species contamination-related factors considered in this study, the feed storing period, source of feed, presence of rodents in the feed storing room, moisture contents of the feed, and feed ingredient types were found to be independently associated with the contamination of aflatoxigenic Aspergillus species. Hence, according to the current finding, feeds stored in rodent-infested rooms were 2.67 times more likely to encounter aflatoxigenic Aspergillus species contamination than those stored in rodent-free rooms. The observed contamination may be attributed to the presence of rodents or insects in the feed (feed storing room) which predispose the feeds to fungal contamination and aflatoxin production by transport primary inoculums as a vector, disseminate spores within the feed, and facilitate colonization and infection by injuring the feedstuffs and increasing the moisture contents of the feed by their by-products [44]. Home-mixed feeds were $27 \%$ less likely exposed to aflatoxigenic Aspergillus species contamination than commercially formulated feeds bought from feed vendors. The findings are in line with the reports of Aliyu in Nigeria [45]. This might be due to that concentrated feeds were more prone to fungal contamination and aflatoxin production during transportation and storing time than feed ingredients because concentrated feeds are crushed and nutrient rich, which creates a suitable condition for fungal contamination and toxin production than feed ingredients. The justification is further supported by the recommendation from the USAID [46] that $23 \%$ aflatoxin production can be reduced by home preparation of feed.

The likelihood of aflatoxigenic Aspergillus species contamination in feeds stored for one to two months was nearly two times that in feeds stored for less than one month, whilst feeds which were stored for more than two months were 2.69 more likely to encounter aflatoxigenic Aspergillus species contamination than feeds that were stored for less than one month. The findings are comparable with the reports in [15] at Hawasa, Ethiopia. The increment of fungal contamination with increasing feed storing time might be due to the increment of the probability of the feed damaged by insects and rodents which increases the moisture contents of the feed that creates conducive environment for fungal contamination and aflatoxin production [47].

Feeds which have low moisture content $(<10 \%)$ were $81 \%$ less likely to encounter aflatoxigenic Aspergillus species contamination than feeds which had medium moisture content (10-14\%), and feeds which had high moisture content $(>14 \%)$ were 1.5 times more likely to be exposed to aflatoxigenic Aspergillus species contamination than feeds which had medium moisture content (10-14\%). The finding is consistent with other previous studies conducted elsewhere $[12,17]$.

The assessment of the awareness on fungal contamination and aflatoxin production in feeds revealed levels of 34.4 and 20.3 percent, respectively, among farm owners, factory managers, and workers. From all the respondents, only 32.8 percent applied prevention practice on fungus contamination. The result is higher than $10 \%$ knowledge and $30 \%$ prevention practice in Addis Ababa [15]. The observed better knowledge and prevention practice against fungal contamination and aflatoxin in feed compared to previous studies might be due to increased access to information on aflatoxin and its health impacts from different medias which create awareness about fungal contamination and effects of aflatoxin in feed and animal products presently than in the past.

\section{Conclusions}

The magnitudes of aflatoxigenic Aspergillus species in poultry feed were found high, and A. flavus is the more frequently isolated aflatoxigenic Aspergillus species than A. parasiticus. Fungal contamination in poultry feed was associated with the presence of rodents in the feed storing room, longer storing period, and high moisture contents of the feed. The highest rate of fungal contamination was observed in maize followed by soya bean and nug cake due to high soluble sugar in maize and high lipid content in soya bean and nug cake, whereas the lowest rate was found in wheat bran associated with its low moisture content and poor nutritional composition as compared with others. As a result of cleaning, sorting, and shorter storing period of feed, lower rate of aflatoxigenic Aspergillus species contamination was observed in home-mixed (farm) feeds than commercially formulated ones bought from feed vendors. A maximum mean aflatoxin level was measured in soya bean, and a minimum mean aflatoxin level was measured in wheat bran. Nearly one-third of the feeds showed the aflatoxin level beyond the maximum FDA permissible level. Generally, biological, environmental, and feed-related factors were found to be key determinant factors associated with the contamination of aflatoxigenic Aspergillus species in poultry feeds. The knowledge levels and adapted prevention 
practices about fungal contamination and aflatoxin production among poultry industry stakeholders were very low.

In line with the abovementioned conclusion, the following recommendations are forwarded:

(i) Awareness creation about the impact of aflatoxin in poultry health and production, together with prevention methods, should be extended to all stakeholders along the poultry value chain

(ii) The farm owners should be encouraged to prepare their poultry feeds by themselves rather than buying commercially prepared concentrated feeds in bulk from feed vendors

(iii) The farm owners should dry the feeds and feed ingredients well before storage and avoid long periods of feed storage

(iv) Feed storing room should be free of insects and rodents, well ventilated, and cleaned before restocking the feed, and the first in first out principle of the feed utilization should be implemented

(v) Surveillance and scheduled aflatoxin monitoring programs should be implemented in poultry farms and commercial poultry feed processing factories

(vi) There have to be country-based set standards on maximum aflatoxin permissible limit in poultry feeds

(vii) Extension programmes targeting farm owners, managers, and workers about knowledge of fungal contamination and aflatoxin in feed need be enhanced

\section{Data Availability}

The raw data used to support the findings of this study are available from the corresponding author upon request.

\section{Conflicts of Interest}

The authors declare no conflicts of interest.

\section{Acknowledgments}

The authors would like to thank Haramaya University for full financial supports of the research expense.

\section{References}

[1] M. Abrar, F. M. Anjum, M. S. Butt, M. A. Randhawa, and S. K. Farhan, "Aflatoxin biosynthesis, occurrence, toxicity and remedies," Food Science and Nutrition, vol. 4, no. 53, pp. 862-874, 2013.

[2] S. M. Herzallah, "Determination of aflatoxins in eggs, milk, meat and meat products using HPLC fluorescent and UV detectors," Food Chemistry, vol. 114, no. 3, pp. 1141-1146, 2009.

[3] E. T. Ejioffor, A. C. Mgbeahuruike, N. O. Okoroafor, and C. J. Aronu, "Aflatoxigenic fungi in Nigerian poultry feeds:," International Journal of Livestock Production, vol. 308-317, 2018.
[4] M. A. Anjum, S. H. Khan, A. W. Sahota, and R. Sardar, "Assessment of AFB1 in commercial poultry feed and feed ingredients," Journal of Animal and Plant Sciences, vol. 22, no. 2, pp. 268-272, 2012.

[5] K. E. Akande, M. M. Abubakar, T. A. Adegbola, and S. E. Bogoro, "Nutritional and health implications of mycotoxins in animal feeds: a review," Pakistan Journal of $\mathrm{Nu}$ trition, vol. 5, no. 5, pp. 398-403, 2006.

[6] B. Noreddine, "Chronic and acute toxicities of aflatoxins. Mechanism of action," International Journal of Environmental Research and Public Health, vol. 17, no. 2, p. 423, 2020.

[7] S. M. Herzallah, "Aflatoxin B1 residues in eggs and flesh of laying hens fed aflatoxin B1 contaminated diet," American Journal of Agricultural and Biological Sciences, vol. 8, no. 2, pp. 156-161, 2013.

[8] A. Ayalew, H. Fehrmann, J. Lepschy, R. Beck, and D. Abate, "Natural occurrence of mycotoxins in staple cereals from Ethiopia," Mycopathologia, vol. 162, no. 1, pp. 57-63, 2006.

[9] D. Gizachew, B. Szonyi, A. Tegegne, J. Hanson, and D. Grace, "Aflatoxin contamination of milk and dairy feeds in the Greater Addis Ababa milk shed, Ethiopia," Food Control, vol. 59, pp. 773-779, 2016.

[10] CSA, "Central statistics agency, Ethiopia," 2015.

[11] M. J. Ibrahim, J. Kabir, C. N. Kwanashie, M. T. Salawudeen, and Z. Joshua, "Occurrence of mycotoxigenic fungi in poultry feeds at live-bird markets, Zaria, Nigeria," Sokoto Journal of Veterinary Sciences, vol. 15, no. 4, pp. 53-59, 2017.

[12] J. Kana, B. Gnonlonfin, J. Harvey et al., "Assessment of aflatoxin contamination of maize, peanut meal and poultry feed mixtures from different agroecological zones in Cameroon," Toxins, vol. 5, no. 5, pp. 884-894, 2013.

[13] I. Rodrigues, J. Handl, and E. M. Binder, "Food additives and contaminants. Mycotoxins occurrence in feeds and feed ingredients in Africa," Food and Conta, vol. 4, no. 3, pp. 168-179, 2011.

[14] R. Mesfin, G. Assef, and F. Assefa, "Determination of aflatoxin in dairy feeds and milk in selected areas of Ethiopia," Journal of Food Engineering, vol. 17, no. 3, pp. 286-299, 2018.

[15] M. Fikere, E. Getu, and T. Bbekele, "Level of aflatoxin in dairy cattle feeds and assesses knowledge and practice around Addis Ababa, Ethiopia," M.Sc. thesis, 2017.

[16] Association of American Food control officials, Feed Inspector's Manual, Association of American Feed Control, Inspection and Sampling Committee, USA, 5th edition, 2014.

[17] N. Sahar, S. Arif, Q. Afzal, S. Aman, J. Ara, and M. Ahmed, "Moisture content and its impact on aflatoxin levels in red chillies," Food Addit \& Contam., vol. 13, no. 2, p. 23, 2014.

[18] Mycology-Critique, Mycology Proficiency Testing Program, pp. 7-15, Wadsworth Center, State Department of Health, New York, NY, USA, 2004.

[19] G. C. James and S. Natalie, Microbiology. A Laboratory Manual, pp. 211-223, Benjamin/Cummings Publishing Company, Redwood City, CA, USA, 2001.

[20] B. A. Sabry, A. S. Hathout, A. Nooh, S. E. Aly, and M. G. Shehata, "The prevalence of aflatoxin and Aspergillus parasiticus in Egyptian Sesame seeds," International Journal of Chem Tech Research, vol. 9, pp. 308-319, 2016.

[21] H. K. Abbas, W. T. Shier, and M. A. Weaver, "Cultural methods for aflatoxin detection," Journal of Toxicology Toxin Reviews, vol. 23, no. 3, pp. 295-315, 2004.

[22] "Aflatoxin B1 ELISA assay-low matrix. Catalog \# 981BAFL01LM-96," http://www.helica.com. 
[23] J. Muhammed, "Determination of aflatoxigenic fungi in poultry feeds and AFB1 in fresh and boiled broiler livers in live bird markets in Nigeria," M.Sc. thesis, 2017.

[24] M. K. Saleemi, A. Khan, I. Javed, Z. Hasan, S. Hameed, and M. A. Mehmood, "Occurrence of toxigenic fungi in maize from Pakistan," Pakistan Journal of Botany, vol. 42, no. 1, pp. 427-434, 2012.

[25] S. Mohammed, J. J. E. Munissi, and S. S. Nyandoro, “Aflatoxin M1in raw milk and aflatoxin Blin feed from household cows in Singida, Tanzania," Food Additives and Contaminants: Part $B$, vol. 9, no. 2, pp. 85-90, 2016.

[26] A. Elzupir, M. H. Younis, H. W. Fadul, and S. Elhuss, "Determinations of aflatoxin in animal feed in Khartoum state, Sudan," Journal of Animal and Veterinary Sciences, vol. 8, no. 5, pp. 1000-1003, 2009.

[27] G. Schatzmayr and E. Streit, "Global occurrence of mycotoxins in the food and feed chain: facts and figures," World Mycotoxin Journal, vol. 6, no. 3, pp. 213-222, 2013.

[28] S. Bailly, A. Mahgubi, A. Carvajal-Campos et al., "Occurrence and identification of Aspergillus section flavi in the context of the emergence of aflatoxins in French maize," Toxins, vol. 10, no. 12 , p. $525,2018$.

[29] K. Nishimwe, E. Bowers, J. d. D. Ayabagabo, R. Habimana, S. Mutiga, and D. Maier, "Assessment of aflatoxin and fumonisin contamination and associated risk factors in feed and feed ingredients in Rwanda," Toxins, vol. 11, no. 5, p. 270, 2019.

[30] P. B. Njobeh, M. F. Dutton, A. T. Åberg, and P. Haggblom, "Estimation of multi-mycotoxin contamination in South African compound feeds," Toxins, vol. 4, no. 10, pp. 836-848, 2012.

[31] J. E. Mellon, P. J. Cotty, and M. K. Dowd, "Influence of lipids with and without other cottonseed reserve materials on AFB1 production by A. flavus," Journal of Agricultural and Food Chemistry, vol. 4, no. 2, pp. 1-3, 2000.

[32] J. Liu, L. Sun, N. Zhang et al., "Effects of nutrients in substrates on AFB1 production by A. flavus," BioMed Research International, vol. 1-2, 2016.

[33] O. Sheila, "I151 building an evidence base for assessing and improving multidisciplinary teams," Rheumatology, pp. 1-10, 2016.

[34] F. Stepman, "Scaling-up the impact of aflatoxin research in Africa. The role of social Sciences," Toxins, vol. 10, no. 4, p. 136, 2018.

[35] K. Kannan, S. Supriya, R. Adhithya, and R. Velazhahan, "Aspergillus flavusand aflatoxin contamination of poultry feeds in Tamil Nadu, India," International Journal of Agriculture Environment \& Biotechnology, vol. 7, no. 2, pp. 361366, 2014.

[36] F. Faparusi and E. Alagamba, "Presence of toxigenic Aspergillus spp. in commercial poultry feeds in Nigeria," Journal of Food Quality and Hazards Control, vol. 5, no. 8, pp. 128-133, 2018.

[37] S. Woyeso, A. Mengestu, F. Tadesse, and Y. Chernet, "Quantification of aflatoxin contamination in dairy and beef cattle compound feeds and feeds ingredients in bishoftu and adama district, Ethiopia," M.Sc. thesis, 2019.

[38] A. S. Dhavan and M. R. Choudary, "Incidence of aflatoxins in animal feedstuffs: a decade's scenario in India," Journal of AOAC International, vol. 78, no. 3, pp. 1-5, 1995.

[39] M. Lubna, M. Debnath, and F. Hossaini, "Detection of aflatoxin in poultry feed and Feed materials through Immuno based assay from different poultry farms and feed factories in
Banglade," Bangladesh Journal of Microbiology, vol. 35, no. 1, 2018.

[40] H. Kerstin and M. Charity, "Aflatoxin control and prevention strategies in key crops of sub-Saharan Africa," African Journal of Microbiology Research, vol. 5, no. 5, pp. 459-466, 2011.

[41] S. Iram, S. K. Fareed, M. Chaudhary et al., "Identification of Aspergillus flavus and aflatoxin in home mix layer poultry feed in relation to seasons in Karachi, Pakistan," Tropical Animal Health and Production, vol. 51, no. 6, pp. 1321-1327, 2018.

[42] M. M. Nkosi, A. .C. L.. Safalaoh, and C. Mtegha, "Prevalence and contamination levels of aflatoxins in dairy feeds in Malawi," African Journal of Rural Development, vol. 3, no. 3, pp. 883-894, 2018.

[43] M. A. Alvarado, R. Z. Sanabria, and F. G. Chinchilla, "Aflatoxins in feedstuffs, levels of contamination, prevalence and control strategies," JAVS, vol. 7, no. 4, pp. 66-70, 2017.

[44] G. Avantaggio, F. Quaranta, E. Desidero, and A. Visconti, "Fumonisin contamination of maize hybrids visibly damaged by sesamia," Journal of the Science of Food and Agriculture, vol. 83, pp. 13-18, 2002.

[45] R. Aliyu, M. Abubakar, Y. Yakubu et al., "Prevalence of potential toxigenic Aspergillus species isolated from poultry feeds in Sokoto metropolis," Sokoto Journal of Veterinary Sciences, vol. 14, no. 1, pp. 39-44, 2016.

[46] USAID, "Aflatoxin: a synthesis of the research on health," Agriculture and Trade, 2012.

[47] Z. Ngoko, W. F. O. Marasas, J. P. Rheeder, M. J. Wingfield, and K. F. Carwell, "Fungal infection and mycotoxin contamination of maize in Cameroon," Phytoparasit, vol. 29, no. 4, pp. 1-9, 2001. 